\title{
Shaping of Alginate-Silica Hybrid Materials into Micro- spheres through Vibrating-Nozzle Technology and Their Use for the Recovery of Neodymium from Aqueous Solutions
}

Joris Roosen, ${ }^{\mathrm{a}, \mathrm{b}}$ Judith Pype ${ }^{\mathrm{a}, \mathrm{c}}$ Koen Binnemans ${ }^{* \mathrm{~b}}$ and Steven Mullens ${ }^{\mathrm{a}}$

a Sustainable Materials Management, Flemish Institute for Technological Research (VITO), Boeretang 200, 2400 Mol, Belgium. ${ }^{b}$ Department of Chemistry, KU Leuven, Celestijnenlaan 20oF, P.O. Box 2404, 3001 Heverlee, Belgium. ${ }^{\mathrm{c}}$ Department of Chemistry, University of Antwerp, Universiteitsplein 1, 2610 Wilrijk, Belgium.

SUPPORTING INFORMATION 
Table S1: Fitting parameters $\mathrm{K}$ and $\mathrm{n}$ within the Ostwald-de Waele model for solutions with different concentrations of sodium alginate.

\begin{tabular}{l|cc}
\hline & K & n \\
\hline $\mathbf{1 . 0} \%(\mathbf{w t} / \mathbf{v o l})$ & 0.036 & 0.979 \\
$\mathbf{2 . 0} \%(\mathbf{w t} / \mathbf{v o l})$ & 0.237 & 0.912 \\
$\mathbf{3 . 0} \%(\mathbf{w t} / \mathbf{v o l})$ & 1.040 & 0.836 \\
$\mathbf{4 . 0} \%(\mathbf{w t} / \mathbf{v o l})$ & 3.028 & 0.778
\end{tabular}

Table S2: Experimental parameters for vibrational droplet coagulation experiments

\begin{tabular}{c|c|c}
\hline & $\begin{array}{c}\text { Pure alginate } \\
\text { (batch 1) }\end{array}$ & $\begin{array}{c}\text { Alginate- } \\
\text { M600 } \\
\text { (batch 2) }\end{array}$ \\
\hline $\begin{array}{c}\text { Nozzle size } \\
(\mu \mathrm{m})\end{array}$ & 400 & 400 \\
$\begin{array}{c}\text { Pressure } \\
(\text { mbar) }\end{array}$ & 780 & 750 \\
$\begin{array}{c}\text { Amplitude } \\
(\mathrm{mV})\end{array}$ & 2000 & 3500 \\
Frequency (Hz) & 750 & 350 \\
\hline
\end{tabular}

Table S3: Mean diameters, corresponding shrink factors and Feret ratios for microspheres dried by different methods.

\begin{tabular}{c|c|c|c|c}
\hline \multirow{2}{*}{ Drying technique } & Material & $\begin{array}{c}\text { Diameter } \\
(\mu \mathrm{m})\end{array}$ & $\begin{array}{c}\text { Shrink } \\
\text { percentage (\%) }\end{array}$ & Feret ratio \\
\hline \multirow{2}{*}{ Dried to the air } & Alginate-TMOS & $549 \pm 38$ & 30 & $0.89 \pm 0.04$ \\
& Alginate-M600 & $399 \pm 36$ & 64 & $0.93 \pm 0.03$ \\
\hline \multirow{2}{*}{ Freeze-dried } & Alginate-TMOS & $595 \pm 45$ & 24 & $0.85 \pm 0.06$ \\
& Alginate-M600 & $846 \pm 48$ & 23 & $0.87 \pm 0.04$ \\
\hline Supercritical $\mathrm{CO}_{2}$ & Alginate-TMOS & $692 \pm 35$ & 11 & $0.90 \pm 0.04$ \\
dried & Alginate-M600 & $839 \pm 35$ & 24 & $0.94 \pm 0.02$
\end{tabular}




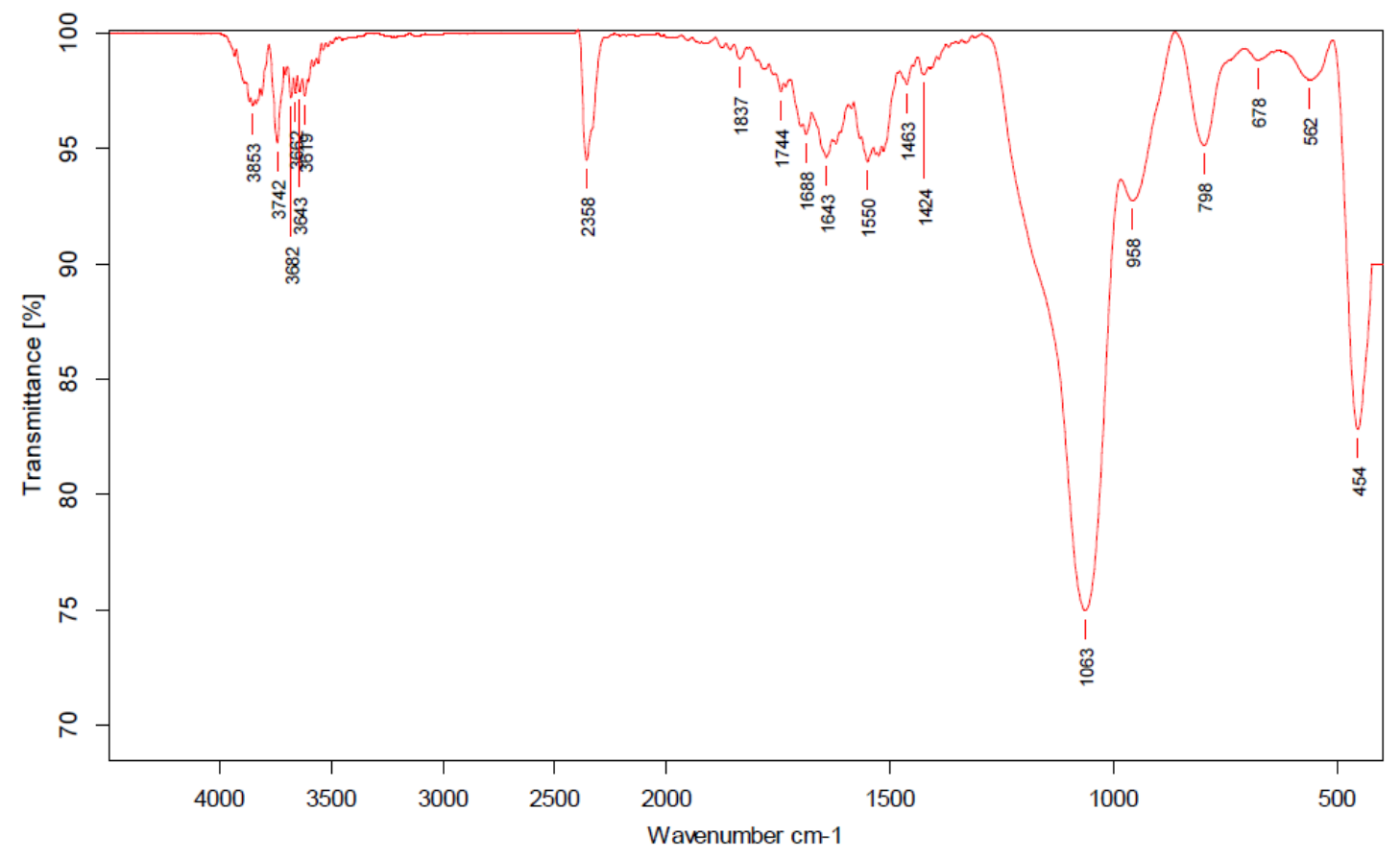

Figure S1: FT-IR spectrum of alginate-TMOS microspheres.

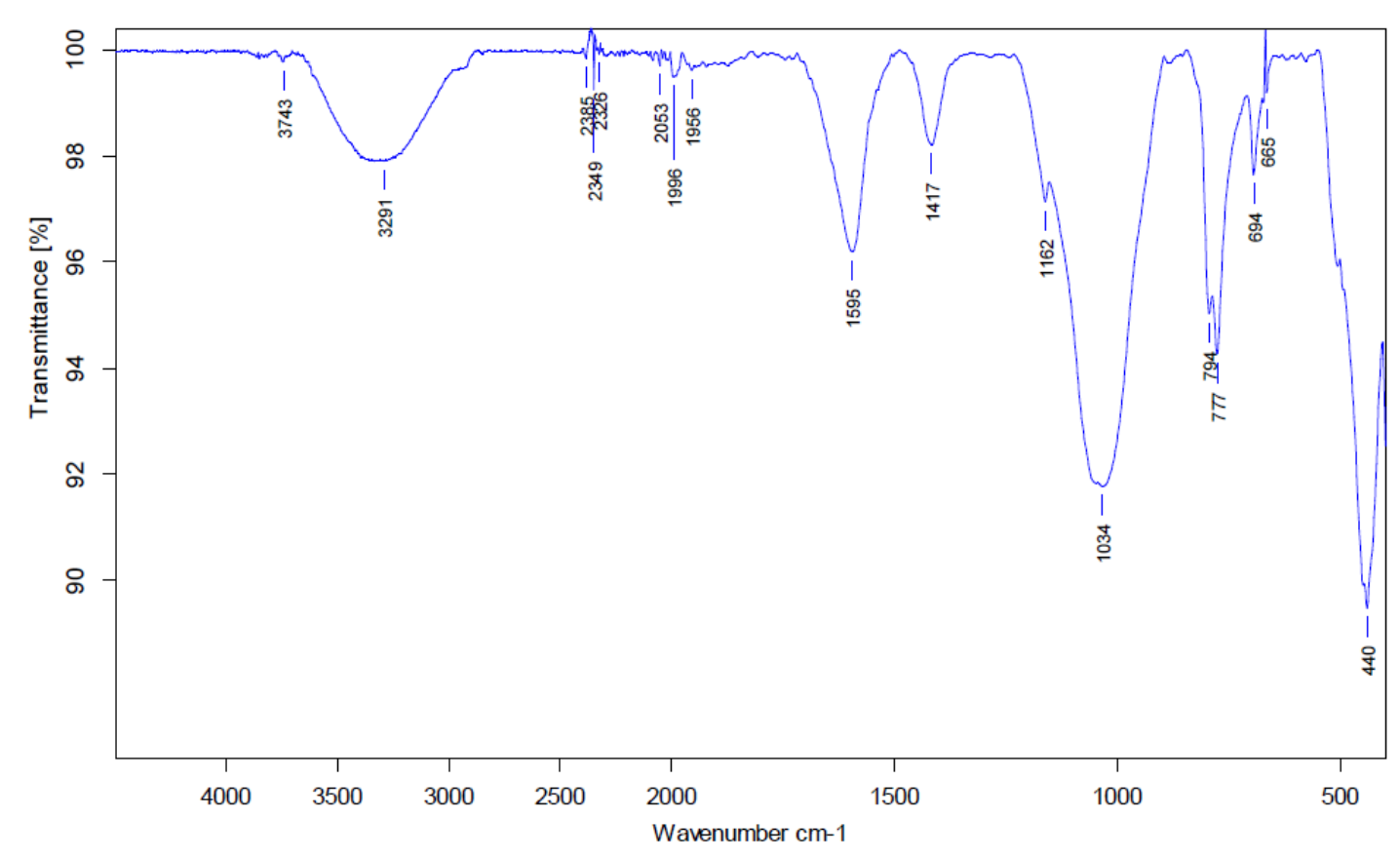

Figure S2: FT-IR spectrum of alginate-silica M600 microspheres. 


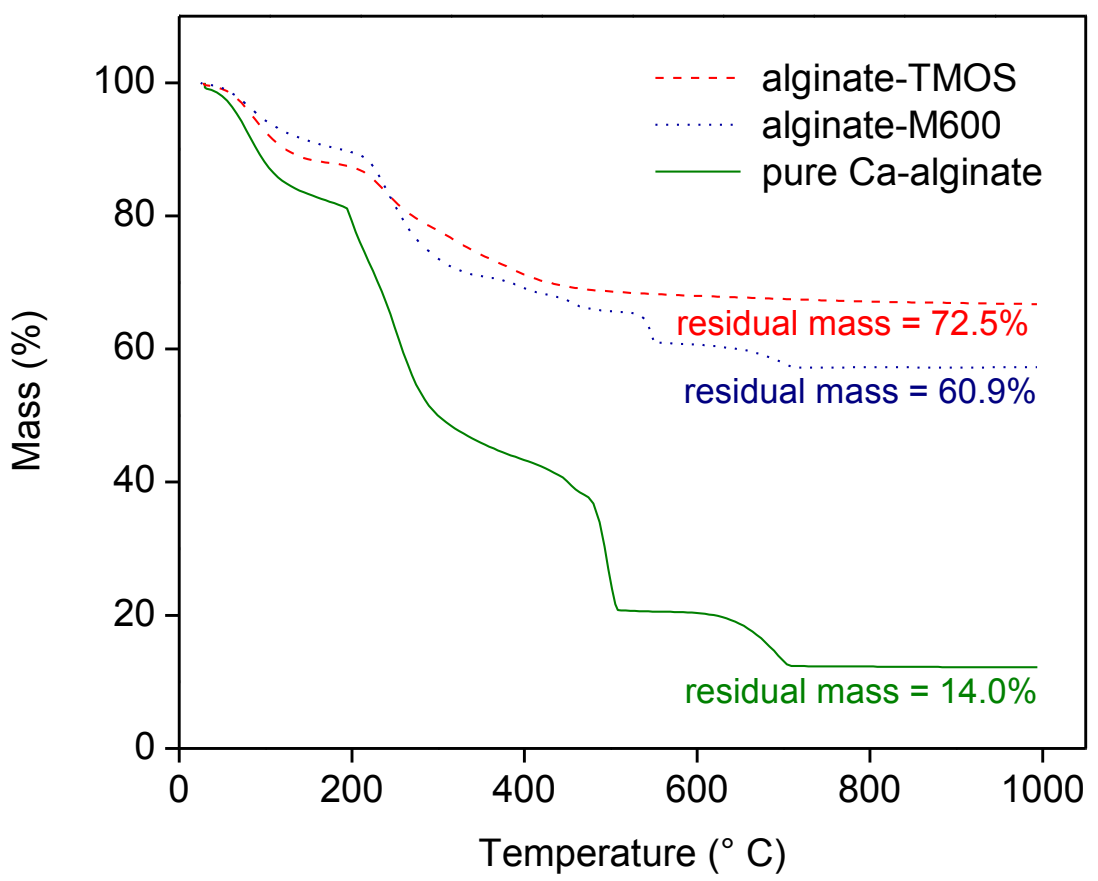

Figure S3: TGA traces of pure calcium alginate, alginate-TMOS, and alginate-silica M600 microspheres (air-dried) and corresponding residual masses (corrected for remaining water content).

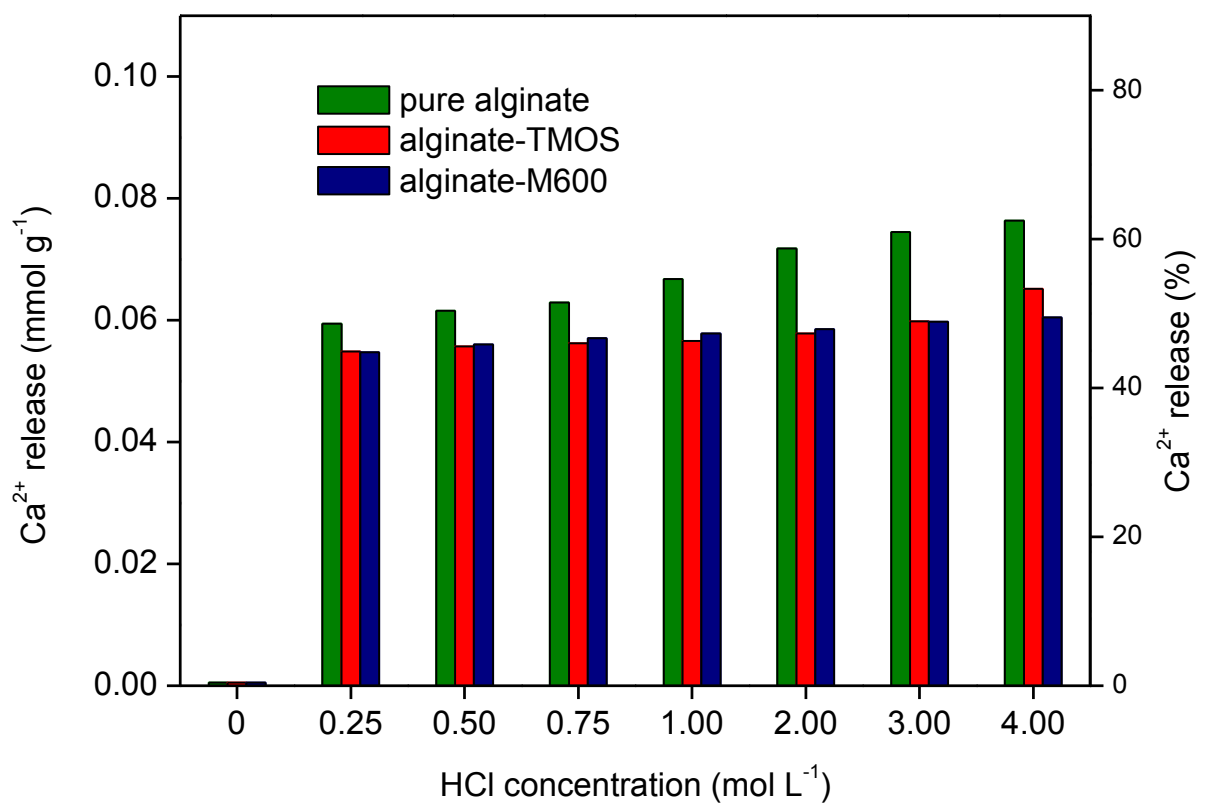

Figure S4: $\mathrm{Ca}^{2+}$ release from alginate(-silica) microspheres (wet) after first contact with solutions of increasing $\mathrm{HCl}$ concentration. 


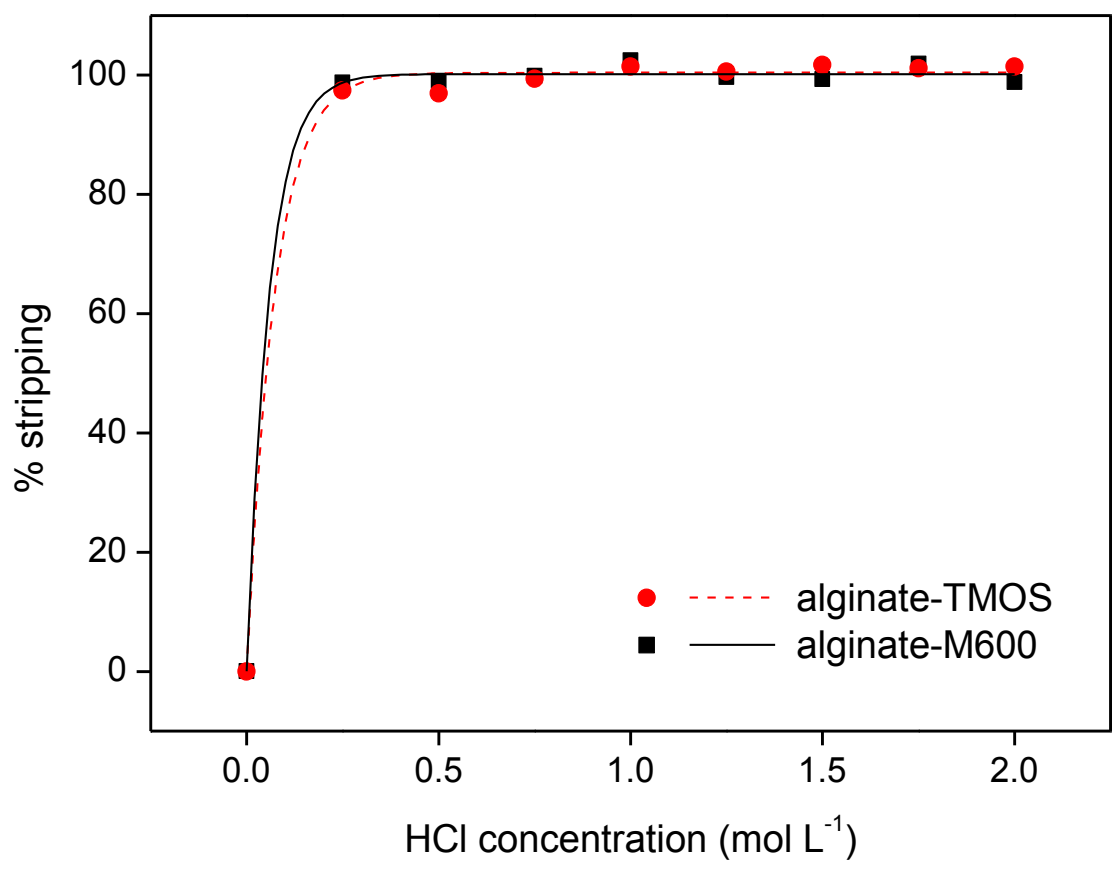

Figure S5: Stripping percentage of alginate-TMOS and alginate-silica M600 microspheres, loaded with $\mathrm{Nd}(\mathrm{III})$, as a function of the $\mathrm{HCl}$ concentration. 